\title{
Determination of the Loading Mode Dependence of the Proportionality Parameter for the Tearing Energy of Embedded Flaws in Elastomers under Multiaxial Deformations
}

\author{
R.J. Windslow ${ }^{1}$, T.W. Hohenberger ${ }^{2}$, and J.J.C. Busfield ${ }^{3}$
}

\begin{abstract}
In this paper, the relationship between the tearing energy and the far field cracking energy density (CED) is evaluated for an embedded penny shaped flaw in a 3D elastomer body under a range of loading modes. A 3D finite element model of the system is used to develop a computational based fracture mechanics approach which is used to evaluate the tearing energy at the crack in different multiaxial loading states. By analysing the tearing energy's relationship to the far field CED, the proportionality parameter in the CED formulation is found to be a function of stretch and biaxiality. Using a definition of biaxiality that gives a unique value for each loading mode, the proportionality parameter becomes a linear function of stretch and biaxiality. Tearing energies predicted through the resulting equation show excellent agreement to those calculated computationally.
\end{abstract}

Keyword Fracture $\cdot$ Elastomer $\cdot$ Cracking energy density $\bullet$ Tearing energy $\bullet$ Biaxiality $\bullet$ Multiaxial

\footnotetext{
${ }^{1}$ R.J. Windslow

Schlumberger, Ltd., 14910 Airline Road, Rosharon, TX, USA

${ }^{2}$ T.W. Hohenberger

Queen Mary University of London, School of Engineering \& Materials Science, Mile End Road, London, UK

${ }^{3}$ J.J.C. Busfield $(\bowtie)$

Queen Mary University of London, School of Engineering \& Materials Science, Mile End Road, London, UK

Email: j.busfield@qmul.ac.uk
} 


\section{Content}

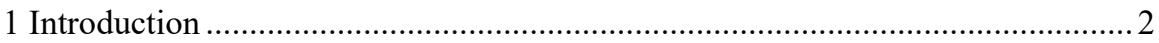

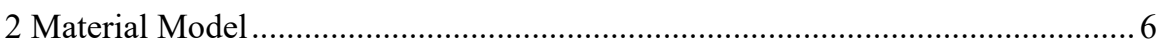

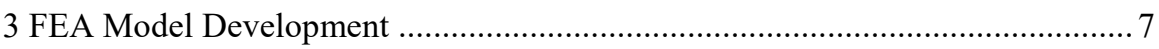

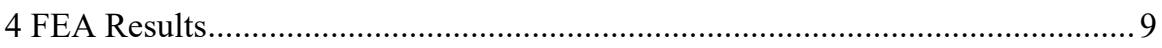

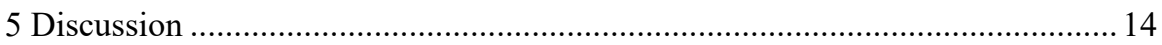

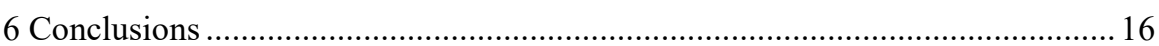

\section{Introduction}

Researchers during the early $20^{\text {th }}$ century suggested that stress concentrations at flaws were the root cause of fracture. However, analytical modelling found that the maximum stress of an elliptical crack approached infinity (a physical impossibility) as the radius of the crack tip tended toward zero. ${ }^{1}$ To overcome this problem, Griffith proposed evaluating the local energy field at the crack tip rather than the more complex stress field. ${ }^{2}$ Griffith's premise was that the release of strain energy as a crack propagated was equivalent to the surface energy required to form the new fracture surfaces. Therefore, a crack would only propagate if the resulting release of strain energy was in excess of the surface energy requirements to create the new faces. This proved successful for brittle materials but did not translate to elastomers as dissipative effects caused the released strain energy to significantly exceed the surface energy.

Realising the issue, Rivlin and Thomas extended Griffith's approach to make it more applicable to elastomers and other polymers. ${ }^{3}$ This was achieved by introducing a critical energy release rate, above which the crack would grow independent of the test piece geometry. The critical energy release rate, or tearing energy, $T$, is equivalent to the rate of change in strain energy, $U$, of the sample, divided by the increase in area of one of the newly formed fracture surfaces, $A$. Under the assumption that the sample was held at constant length, $l$, the energy contribution due to external work could be negated, Eqn. (1).

$$
T=-\left(\frac{\mathrm{d} U}{\mathrm{~d} A}\right)_{l}
$$

Rivlin and Thomas also showed that the tearing energy could be applied globally to the system or locally about the crack tip. This allowed them to develop analytical solutions to quantify the tearing energy in edge cracked samples when deformed in pure shear and uniaxial tension. One of the key derivations was for the single edge notched tension, SENT, test piece. Owing to the introduction of the crack, the SENT test piece can be divided into three regions: two far field regions under uniform 
deformation and a complex energy region influenced by the crack, Figure 1. Through the use of a proportionality parameter, $k$, Rivlin and Thomas defined the tearing energy available for a given crack length, $c$, and strain energy density, SED or $w$, in the far field region, Eqn. (2).

$$
T=2 k w c
$$

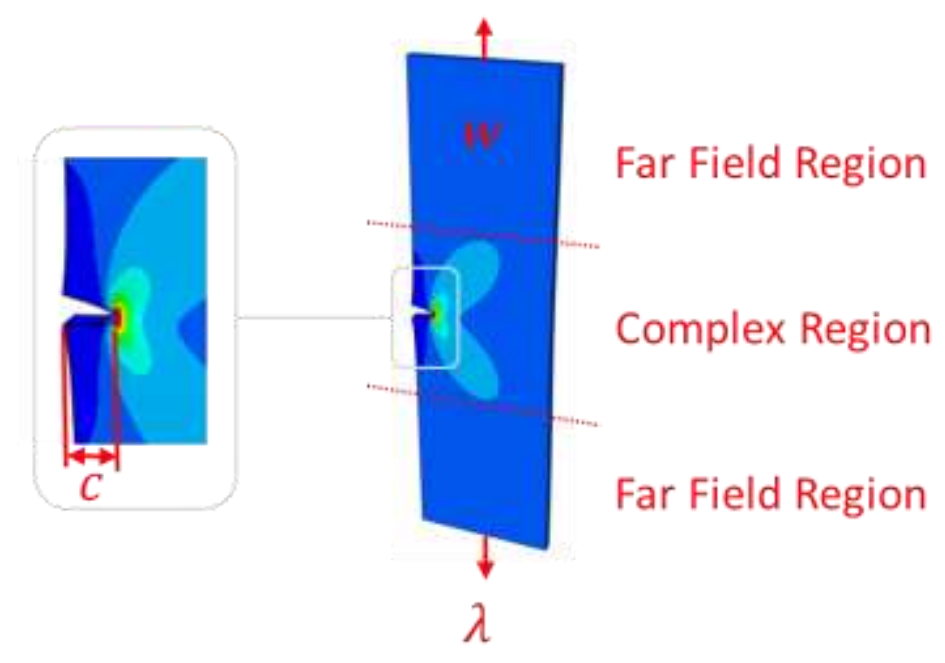

Fig.1. Strain energy density field in a SENT test piece under uniaxial tension

One of the barriers for this approach was suitably defining the proportionality parameter, $k$. Through experimentation, Greensmith showed that it was loosely dependent upon the far field principal stretch, $\lambda$, decreasing from $k=3$ at small strains to $k(\lambda=3)=2 \cdot{ }^{4}$ Later Lindley used finite element analysis (FEA) to define this constant in terms of the principal stretch, Eqn. (3). ${ }^{5}$ His expression broadly agreed with Greensmith's results.

$$
k=\frac{2.95-0.08(1-\lambda)}{\sqrt{\lambda}}
$$

At a similar time, Lake attempted to use symmetry conditions to adapt Rivlin and Thomas' SENT solution to the tearing energy at a centrally positioned flaw in a thin sheet under uniaxial tension. ${ }^{6} \mathrm{He}$ found the proportionality parameter related to the far field principal stretch through $\pi$, Eqn. (4). The value of the proportionality parameter is relatively similar regardless of whether it is expressed through Eqn. (3) or Eqn. (4). Due to this researchers typically use the simpler form, Eqn. (4), for both edge and central cracks. However, it is worth noting that Klüppel and coworkers have published experimental data from SENT test pieces that suggests the factor of 
$\pi$ is too high, so they replaced it with a front factor that ranged from $1.2-3.1 ._{-}^{7} \mathrm{Nev}$ erthelessIn any case, Eqns. (3) and (4)both of these expressions are only suitable when the global system is under uniaxial tension and for small to moderate strains, $1 \leq \lambda \leq 3$.

$$
k=\frac{\pi}{\sqrt{\lambda}}
$$

Alternative expressions to make Eqn. (2) suitable for multiaxial deformations at finite strains have been proposed. Yeoh observed that under far field tension an embedded penny shaped flaw became elliptical. $\stackrel{8}{ }$ He deduced that for a flaw of initial radius (that is, half-length), $c$, which under deformation formed an ellipse of length, $2 a^{\prime}$, and height, $2 b^{\prime}$, the proportionality parameter would be a function of the geometry, the SED in the far field region and the Cauchy stress, $\sigma_{\mathrm{y}}$, acting against the flaw opening, Eqn. (5). The model showed good agreement against tearing energy values calculated using computational based fracture mechanics up to $100 \%$ strain in uniaxial and planar tension; however, it provided a poor fit in equibiaxial tension.

$$
k=\frac{\sigma_{\mathrm{y}} \pi b^{\prime}}{4 w c}
$$

An alternative approach has been presented, and gained traction, in which the load mode dependence of the fracture process is accounted for by scaling the far field energy variable to represent only the portion that is actually available for release upon fracture. The approach consequently assumes the proportionality factor is independent of loading mode. ${ }^{-}$The scaled far field energy variable is referred to as the cracking energy density, CED or $w_{\mathrm{c}}$. For a flaw in a linearly elastic material, the CED has been quantified in terms of the stress tensor, $\sigma$, the incremental strain tensor, $\mathrm{d} \boldsymbol{\varepsilon}$, and a unit vector normal to the crack plane, $\vec{r}$.

$$
\mathrm{d} w_{\mathrm{c}}=\left(\vec{r}^{\mathrm{T}} \boldsymbol{\sigma}\right) \cdot(\mathrm{d} \boldsymbol{\varepsilon} \vec{r})
$$

More recently, a large strain formulation for the CED based upon the Ogden hyperelastic model has been derived in terms of the $2^{\text {nd }}$ Piola-Kirchoff principal stresses, $S_{\mathrm{i}}$, the principal stretches, $\lambda_{\mathrm{i}}$, and the orientation, $\theta$, of the void with respect to the principal axes of stress. $\underline{10,11}$

$$
\mathrm{d} w_{\mathrm{c}}=\frac{\lambda_{1} S_{1} \cos ^{2} \theta}{\cos ^{2} \theta+\left(\frac{\lambda_{1}}{\lambda_{2}}\right)^{2} \sin ^{2} \theta} \mathrm{d} \lambda_{1}+\frac{\lambda_{2} S_{2} \sin ^{2} \theta}{\cos ^{2} \theta+\left(\frac{\lambda_{1}}{\lambda_{2}}\right)^{2} \sin ^{2} \theta} \mathrm{d} \lambda_{2}-\frac{\lambda_{1} \lambda_{2}{ }^{3} S_{2} \sin ^{2} \theta}{\cos ^{2} \theta+\left(\frac{\lambda_{1}}{\lambda_{2}}\right)^{2} \sin ^{2} \theta} \mathrm{d} \lambda_{3}
$$

In Eqn. (7), $\lambda_{1}$ and $\lambda_{2}$ are assigned as maximum and mid principal stretches, respectively. The CED is one of the most important breakthroughs in fracture mechanics for elastomers since Rivlin and Thomas' initial paper from 1953. There is 
a large body of work which physically validates the CED as a useful tool for evaluating fracture in elastomers, particularly in fatigue analysis. $\frac{12-15}{1 n}$ these works, fatigue tests have been run on a range of geometries using different loading modes. In each, the CED has been found to be the best current fatigue criterion for unifying cycles to failure from different loading modes into a single Wöhler curve. Nevertheless, there has yet to be a full study which demonstrates that the proportionality parameter, assumed constant in the CED approach, is truly independent of the loading mode, particularly for embedded flaws.

A partial study has been carried out as part of the initial CED paper; however, it did not directly compare the results against pure fracture mechanics. ${ }^{2}$ In the paper, the tearing energy for an embedded flaw under uniaxial, planar and equibiaxial deformations was calculated using the proportionality constant defined by linear elastic fracture mechanics (LEFM) for an axisymmetrical embedded flaw under uniaxial tension. The proportionality constant was then assumed to be independent of loading mode, with the load mode dependence being accounted for through the $\mathrm{CED}$. The predicted tearing energies were then compared against values from a previous paper, - which had used pure fracture mechanics to study the tearing energy under these loading modes. As the earlier paper had evaluated a central crack in a flat sheet, a different problem case, the results were compared by normalising the tearing energy values from the equibiaxial and planar states against the predictions from the uniaxial state. The normalised values from the two cases were in rough, although not exact, agreement and hence formed a good start for evaluating the tearing energy in multiaxial cases. Nevertheless, the work could not directly indicate whether the tearing energy was suitably predicted for the embedded flaw case. It only showed that the tearing energy's load mode dependence was similar between the two cases.

There have been a couple of papers which have used pure fracture mechanics to determine the tearing energy of an embedded flaw using the far field energy. $\frac{16,17}{}$ In both these papers, the tearing energy was represented through Eqn. (8) which is an adaption of Eqn. (2) from its flat sheet form into an expression more suited to an embedded penny shaped crack. Neither of these papers used the CED; rather the SED was used with the proportionality parameter's definition accounting for the load mode dependence. In one of these papers, multiple deformation modes were studied, with proportionality constants being derived for uniaxial and equibiaxial loading. $\frac{16}{}$ In the other paper, only uniaxial deformations were studied. Neither of these studies accounted for the proportionality parameter's dependence on the loading mode in a manner that is broadly general for different loading modes.

$$
T=\frac{3}{2 \pi} k w c
$$

In this paper, the relationship between the tearing energy and the far field CED is evaluated for an embedded penny shaped flaw in a 3D elastomer body under a range of loading modes. A 3D finite element model of the system is developed be- 
fore a computational based fracture mechanics approach is used to evaluate the tearing energy at the crack in a range of multiaxial states. By analysing the tearing energy's relationship to the far field CED, a stretch-dependent proportionality parameter is determined for each loading mode. From this, it is shown that the CED approach does not fully account for the load mode dependence of the proportionality parameter; however, a simple relationship for this parameter is deduced from the biaxiality of each loading mode. To proceed, Eqn. (8) is adapted to replace the SED variable with the CED as shown in Eqn. (9). To differentiate between the proportionality parameters in each equation, a subscript ' $c$ ' is used for the CED variant.

$$
T=\frac{3}{2 \pi} k_{\mathrm{c}} w_{\mathrm{c}} c
$$

\section{Material Model}

The simulations use two carbon black filled elastomers: a nitrile rubber (NBR) and an ethylene-propylene-diene-monomer (EPDM) rubber, both of which were supplied by Clwyd Compounders. The elastomers were characterised at $23^{\circ} \mathrm{C}$ using uniaxial tension dumbbell, uniaxial compression button and rectangular planar tension test piece geometries. The nominal gauge dimensions of the dumbbells were $\underline{25 \mathrm{~mm}} \times \underline{6 \mathrm{~mm}} \times \underline{2 \mathrm{~mm}}$. The nominal diameters and heights of the buttons were $25 \mathrm{~mm}$ and $12.5 \mathrm{~mm}$, respectively The buttons were compressed on their axial surfaces but free to radially expand. The nominal gauge dimensions of the planar test pieces were $150 \mathrm{~mm} \times 15 \mathrm{~mm} \times 2 \mathrm{~mm}$. Each test was carried out at $1 \%$ strain per second. Three repeats were carried out per test with the median profile being used to calibrate hyperelastic material models. The test data were then fitted to $2^{\text {nd }}$-order Ogden hyperelastic functions, wherein the elastomers were treated as fully incompressible, Figure 2. The model's derived fitting parameters, $\mu_{\mathrm{i}}$ and $\alpha_{\mathrm{i}}$, are provided in Table 1. Although it is not shown in the figure, the model's behaviour in equibiaxial tension has been confirmed to be realistic. It is worth noting that Abaqus, the FEA solver used for the modelling work, has a slightly different definition of the Ogden model compared to Ogden's original formulation. $\frac{18}{}$ Abaqus' formulation is given in Eqn. (10). $\mathrm{N}$ is the model order and $\lambda_{\mathrm{i}}$ 's are principal stretches.

$$
w=\sum_{\mathrm{i}=1}^{\mathrm{N}=2} \frac{2 \mu_{\mathrm{i}}}{\alpha_{\mathrm{i}}{ }^{2}}\left(\lambda_{1}{ }^{\alpha_{\mathrm{i}}}+\lambda_{2}{ }^{\alpha_{\mathrm{i}}}+\lambda_{3}{ }^{\alpha_{\mathrm{i}}}-3\right)
$$

Table 1. Second-order Ogden material model parameters for the two elastomers

\begin{tabular}{ccccc}
\hline Elastomer & $\mu_{1} / \mathrm{MPa}$ & $\alpha_{1}$ & $\mu_{2} / \mathrm{MPa}$ & $\alpha_{2}$ \\
\hline $\mathrm{NBR}$ & 0.540 & 3.341 & 1.490 & -0.232 \\
$\mathrm{EPDM}$ & 0.338 & 3.272 & 1.327 & 0.089 \\
\hline
\end{tabular}




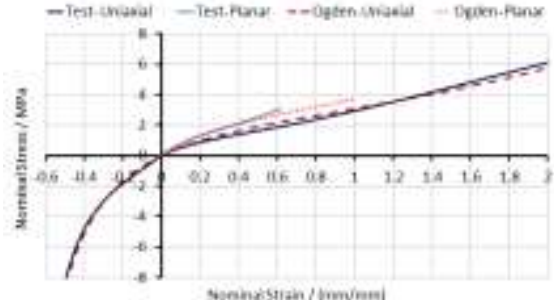

(a)

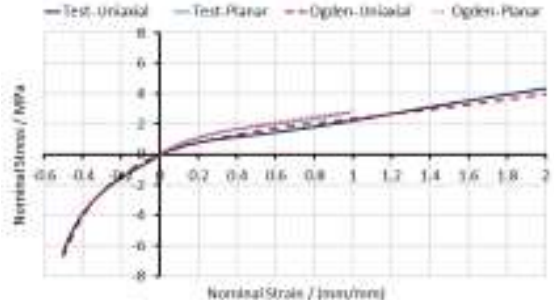

(b)

Fig.2. Fit of the $2^{\text {nd }}$-order Ogden hyperelastic material models to the elastomers' test data for (a) NBR and (b) EPDM

\section{FEA Model Development}

To study the embedded void case, a quartile section of a $2 \mathrm{~mm} \times 2 \mathrm{~mm} \times 2 \mathrm{~mm}$ cube was modelled in Abaqus with a penny shaped crack at its centre. The penny shaped crack was created using the seam interaction function. Symmetry boundary conditions were applied at the internal faces of the quartile section such that it behaved as if part of the whole cube. Displacement boundary conditions were applied to the cube's external faces to impose desired deformation modes. The model was meshed using $1^{\text {st }}$-order hex hybrid elements with enhanced hourglassing control. As the key energy fields were in the complex region around the crack tip, the mesh density was biased towards this region. Mesh convergence found that 37,800 elements formed a suitable mesh density for capturing the strain energy stored in the system. The model is shown in Figure 3.
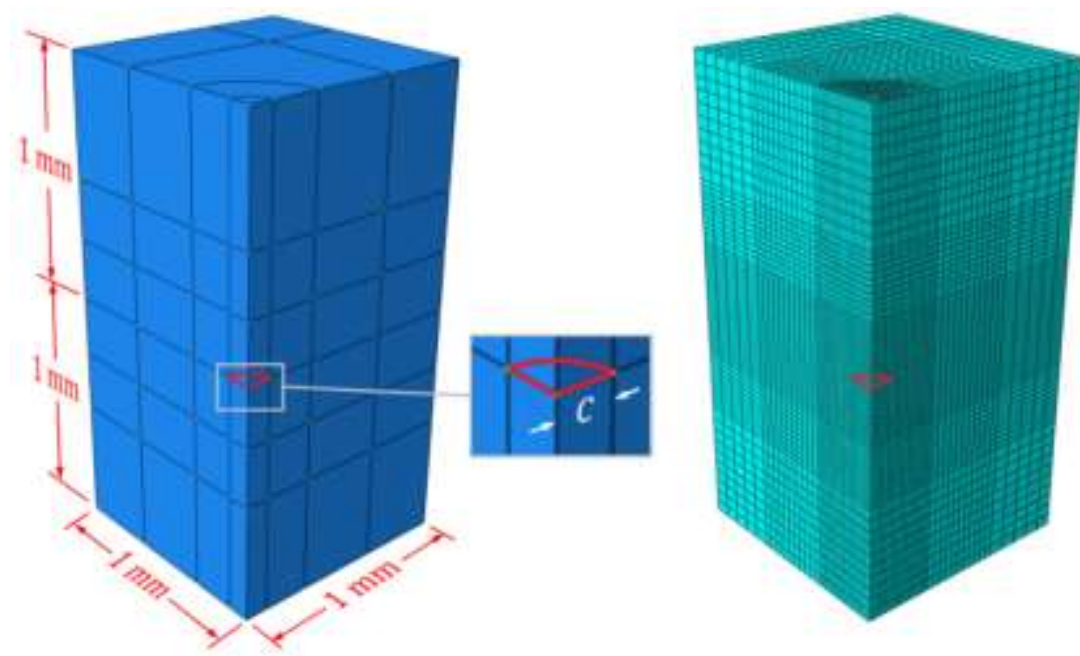

Fig.3. Modelled quartile system with the embedded penny shaped crack of length $c$ 
The computational based Energy Balance fracture mechanics approach was used to evaluate the tearing energy. ${ }^{19}$ This approach is essentially the direct application of Eqn. (1); therefore, it is extremely accurate provided the mesh density is fine enough to capture the strain energy stored in the global system. To apply, the system is modelled through the desired deformation process multiple times, wherein each re-simulation the crack's length has been incrementally increased. This builds a relationship between the strain energy stored in the system with the crack's surface area for a given stretch. The gradient of this plot is the rate at which energy is released by the crack propagating which, as per Eqn. (1), is the tearing energy. In this work, for each loading mode modelled, the unit cube was re-simulated with the crack's radius, $c$, varying from $0.1 \mathrm{~mm}$ to $0.3 \mathrm{~mm}$ in $0.025 \mathrm{~mm}$ increments. As only a quarter of the system was modelled, in each case the strain energy was multiplied by 4 to determine the strain energy in the system as a whole before the tearing energy was calculated.

The tearing energy was evaluated for 6 loading modes. These were the typical loading modes - uniaxial, planar and equibiaxial - and 3 intermediate biaxial states somewhere between planar and equibiaxial deformation. In each of the loading modes, the crack would undergo a Mode I 'opening' type fracture. As such, simple shear was not studied here. A helpful approach that can be used to characterise the loading modes of incompressible elastomers is to define a measure of biaxiality, $B$, such that $\lambda_{2}=\lambda_{1}{ }^{B}$ and $\lambda_{3}=\lambda_{1}{ }^{-(1+B)}$ ?

$$
B=\frac{\log \lambda_{2}}{\log \lambda_{1}}
$$

For the current purposes, there is a slight issue with this definition of the biaxiality in that it does not account for the load mode equivalencies exhibited by elastomers. As shown in Table 2, three different biaxiality values occur with the same loading mode, depending on the direction of loading. More concerning, however, is that the same biaxiality value is achieved for uniaxial tension in the $\lambda_{1}$ axis as equibiaxial tension in the $\lambda_{2}$ and $\lambda_{3}$ axes. Other definitions of biaxiality have been proposed, $\underline{\underline{20}}$ but the authors chose to resolve this issue with a subtle adjustment of Eqn. (11). By referring to the maximum and minimum principal stretch components, $\lambda_{\max }$ and $\lambda_{\min }$ in Eqn. (12), each loading mode has a unique biaxiality, regardless of the direction of loading as shown in Table 2. This arrangement assumes that the crack is initially perpendicular to the max stretch direction, which is typically the orientation that generates the largest tearing energy in literature. $\frac{10}{10}$

$$
B=\frac{\log \lambda_{\min }}{\log \lambda_{\max }}
$$


Table 2. Comparison of the biaxiality definitions

\begin{tabular}{cccccc}
\hline \multirow{2}{*}{ Loading Modes } & \multicolumn{3}{c}{ Principal Stretches } & \multicolumn{2}{c}{ Biaxialities, $B$} \\
& $\lambda_{1}$ & $\lambda_{2}$ & $\lambda_{3}$ & Eqn. (11) & Eqn. (12) \\
\hline Equibiaxial $\left(\lambda=\lambda_{1}=\lambda_{2}\right)$ & 2.0 & 2.0 & 0.25 & 1.0 & -2.0 \\
Equibiaxial $\left(\lambda=\lambda_{2}=\lambda_{3}\right)$ & 0.25 & 2.0 & 2.0 & -0.5 & -2.0 \\
Equibiaxial $\left(\lambda=\lambda_{1}=\lambda_{3}\right)$ & 2.0 & 0.25 & 2.0 & -2.0 & -2.0 \\
Uniaxial Tension $\left(\lambda=\lambda_{1}\right)$ & 2.0 & 0.707 & 0.707 & -0.5 & -0.5 \\
\hline
\end{tabular}

The biaxialities of the loading modes modelled in this work are given in Table 3. The CED/SED values provided were calculated using the small strain CED solution given by Zine et al., Eqn. (13), and are used purely to illustrate the load mode dependence of the energy release. $\frac{10}{-}$ In the equation $v$ is Poisson's ratio.

$$
\frac{w_{c}}{w}=\frac{(B v+1) \cos ^{2} \theta+\left(B^{2}+B v\right) \sin ^{2} \theta}{B^{2}+2 B v+1}
$$

As the fracture process is studied to high strains, the finite strain formulation provided in Eqn. (7) is used to numerically calculate the CEDs in this study. It should be noted that the redefinition of the biaxiality does not alter the CED/SED ratio for a given loading mode; rather the values correlate with previous findings for the same loading modes.,$\stackrel{9,10}{ }$

Table 3. Biaxialities of the loading modes modelled

\begin{tabular}{ccc}
\hline Loading Mode & Biaxiality, $B$ & CED/SED \\
\hline Uniaxial & -0.50 & 1.000 \\
Planar & -1.00 & 1.000 \\
Complex 1 & -1.25 & 0.857 \\
Complex 2 & -1.50 & 0.714 \\
Complex 3 & -1.75 & 0.595 \\
Equibiaxial & -2.00 & 0.500 \\
\hline
\end{tabular}

\section{FEA Results}

The energy fields in the deformed cubes are shown in Figure 4 for the different loading modes. The flaw deformed to different extents depending on which loading mode was used, and maximum strain governed the stability of the model. As such the uniaxial and planar deformations were studied up to $\lambda_{\max }=3.0$, the three intermediate states were studied up to $\lambda_{\max }=2.5$ and the equibiaxial case was studied up to $\lambda_{\max }=2.0$. In each case the tearing energy was evaluated at 10 evenly spaced increments over the deformation. 


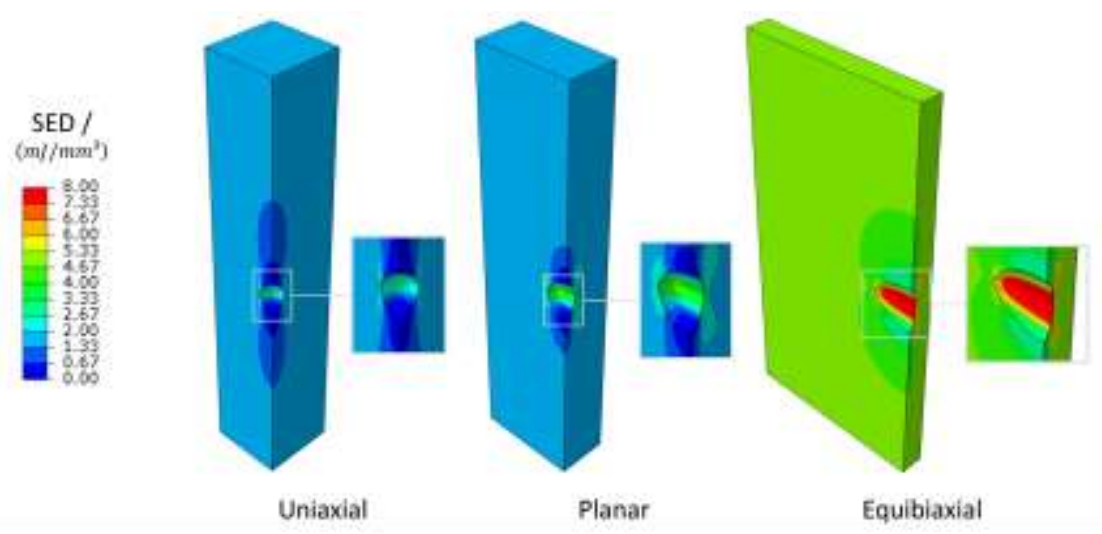

Fig.4. SED fields in deformed cubes under uniaxial, planar and equibiaxial loading at a stretch of

$$
\lambda_{\max }=2
$$

For each loading mode, the strain energy was plotted against the surface area of one face of the undeformed crack under constant stretch. These profiles were fitted with $4^{\text {th }}$-order polynomials, which were then differentiated to give the tearing energy for the given crack area, Figure 5a. Plotting the tearing energy against the crack's radius for a range of stretches results in the expected linear relationship between the crack's length and tearing energy, Figure 5b.

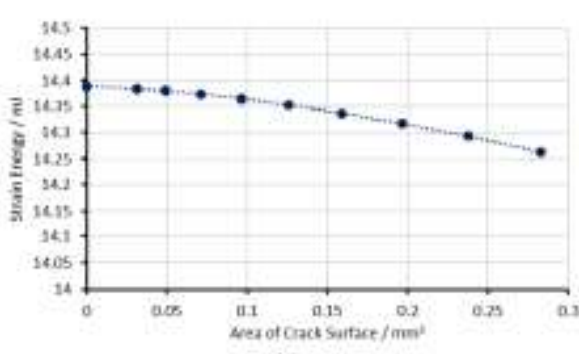

(a)

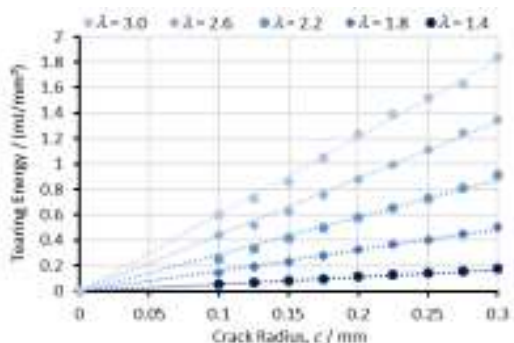

(b)

Fig.5. (a) Energy release in NBR as the crack grows during uniaxial extension with $\lambda=2$; (b) Effect of stretch on the tearing energy of NBR during uniaxial extension

Once the tearing energy was known for the given deformations, the proportionality parameters could be determined by rearranging the tearing energy equations. For each case, two sets of proportionality parameters were calculated, one based on Eqn. (8) where the tearing energy was defined in terms of the SED, and another defined in terms of the CED, Eqn. (9). These will be referred to as $k$ and $k_{\mathrm{c}}$, respectively, and are defined through Eqns. (14a) and (14b).

$$
k=\frac{T}{c} * \frac{1}{w} * \frac{2 \pi}{3}
$$




$$
k_{\mathrm{c}}=\frac{T}{c} * \frac{1}{w_{\mathrm{c}}} * \frac{2 \pi}{3}
$$

For both equations, the $T / c$ term is the gradient formed by plotting $T$ vs. $c$ and then fitting a linear trendline to the data points, Figure $5 \mathrm{~b}$. For each of the loading modes, the proportionality parameter was determined for each stretch value that was simulated, allowing careful study of the parameter's load mode dependence. This dependence was compared in terms of the SED and CED, Figure 6, which reveals an important result. Although the initial purpose of the CED was to remove the load mode dependence of the proportionality parameter for a given SED, the figures suggest that this is not the case; rather, CED causes the profiles to actually spread. It should be noted that as these profiles are plotted against the SED and CED, the power terms of the chosen strain energy function are likely to influence how the profiles stretch along the horizontal axis. The hyperelastic model dictates the rate of energy storage with stretching and biaxiality; as such, the correlation of the profiles may vary when different strain energy functions or fitting parameters are used.

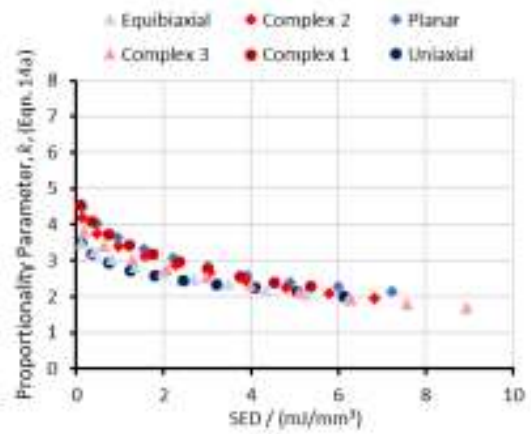

(a)

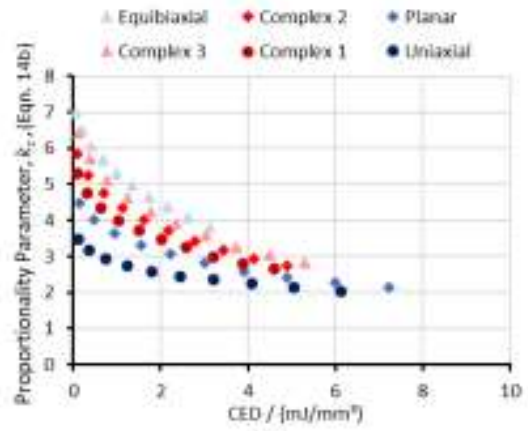

(b)

Fig.6. Relationship between the proportionality parameter and the (a) SED and (b) CED for NBR

In Figure 7 the proportionality parameters' dependence on the stretch is compared for both cases, SED and CED. The uniaxial and planar responses are identical because $w_{\mathrm{c}} / w=1$ for these load cases. The proportionality parameters calculated for the uniaxial and equibiaxial cases, defined through the SED, show very good agreement with previous research. $\frac{16,17}{17}$ For the uniaxial case at low strains, $k=4$ which corresponds to the LEFM solution whilst the overall profile matches those found previously. The equibiaxial data also corresponds with that of Gough and Muhr, who found the SED proportionality parammeter varied from $k(\lambda=1)=4$ to $k(\lambda=1.6)=2 \cdot \frac{16}{}$ Again, when plotting the proportionality parameter in terms of the stretch, using the CED heightens the load mode dependence of the proportionality parameter. Nevertheless, this may actually be beneficial as it causes the separate profiles to all converge towards $k=2$ at $\lambda \geq 3$. This does not occur with the SED, particularly for the equibiaxial case. 


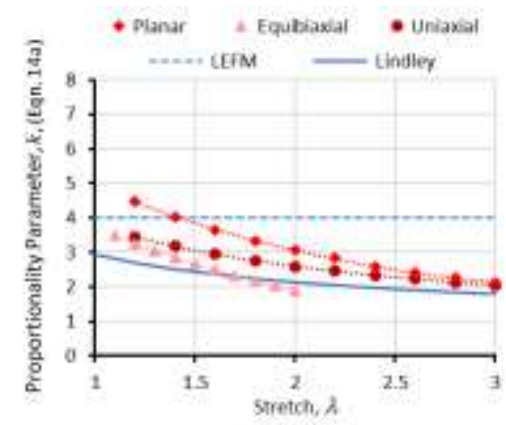

(a)

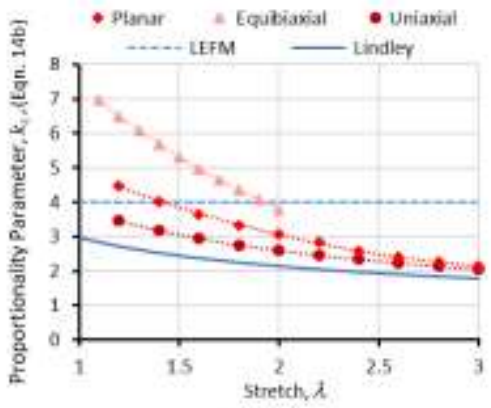

(b)

Fig.7. Relationship between the proportionality parameter and the stretch when calculated using the (a) SED and (b) CED in NBR

The convergence of the proportionality parameter for the CED case is similar to the SENT case wherein Greensmith noted that it decreased from $k=3$ at small strains to $k(\lambda=3)=2$, as shown by the Lindley profile plotted in the figures. ${ }^{5,6}$ In both cases, SENT and embedded flaw, the tearing energy appears to be directly related to the CED at $\lambda \geq 3$; that is, $k_{\mathrm{c}}$ tends toward a constant value as strain increases. It is only at lower strains, $\lambda \leq 3$, that $k_{\mathrm{c}}=k_{\mathrm{c}}(\lambda)$ (as opposed to $k_{\mathrm{c}}=$ constant) is required to properly proportion the far field CED to the tearing energy. As noted by Gough and Muhr, $\frac{16}{1}$ it appears that reducing the biaxiality (or equivalently, tending towards an equibiaxial loading state) increases the proportionality parameter's dependence on the stretch.

For central and edge flaws in flat sheets, both Lake and Lindley found that the proportionality parameter varied with the inverse of the square root of the stretch ratio. ${ }^{6,7}$ In the present work, this is assumed for the embedded flaw as well. As such, the proportionality parameter calculated using the CED is plotted against this relation. There is a complication in determining which stretch component to use, particularly for the biaxial deformation states. Considering that in each case the fracture process is being driven by $\lambda_{\text {max }}$, it is chosen for the abscissa in Figure 8 for the different loading modes and elastomers.

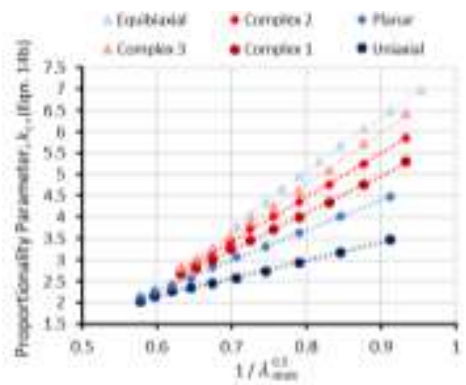

(a)

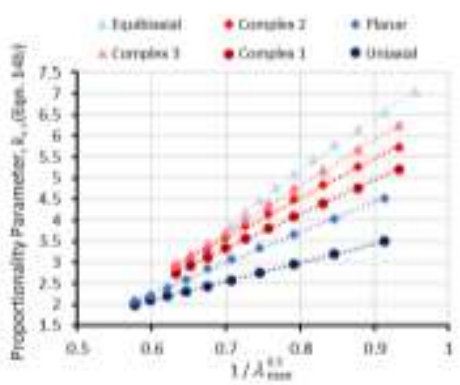

Fig.8. Relationship between the CED proportionality parameter and the inverse square root of the max stretch term in (a) NBR and (b) EPDM 
For both elastomers, the profiles form identical linear relationships for the given loading modes. The interesting point is that the ordering of the profiles, in terms of their gradients and y-intercepts, is related to the loading modes' biaxialities. The profiles become steeper as the biaxiality decreases, but all converge to the same point at $\lambda_{\max }=3$ (that is, $\lambda_{\max }^{-0.5} \approx 0.58$ ). As such, the proportionality parameter forms a linear relationship to the inverse square root of the max principal stretch, through gradient, $m$, and y-intercept, $b$.

$$
k_{\mathrm{c}}=\frac{m}{\sqrt{\lambda_{\max }}}+b
$$

Plotting the gradient, $m$, and $y$-intercept, $b$, of these profiles against the biaxiality of the loading modes yields two linear relationships, Figure 9. This linear relationship would not be seen if the tearing energy was represented through the SED rather than the CED. From these two profiles, a new general definition of the proportionality parameter can be defined, for use with the CED, in terms of the loading mode biaxialities.

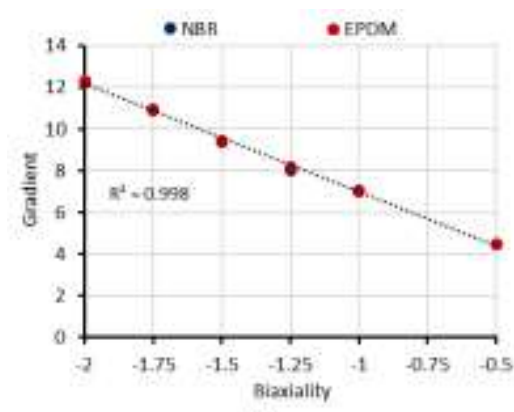

(a)

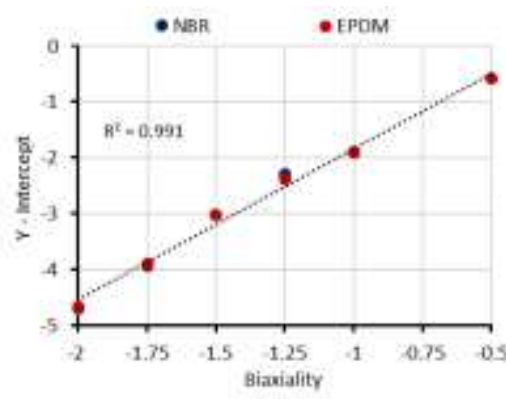

(b)

Fig.9. Relationship between the biaxiality and the (a) gradients and (b) y-intercepts of the profiles in Figure 8

The gradient and y-intercept themselves form linear relationships to the biaxiality, resulting in:

$$
\begin{gathered}
m=m_{\mathrm{m}} B+b_{\mathrm{m}} \\
b=m_{\mathrm{b}} B+b_{\mathrm{b}}
\end{gathered}
$$

Here, $m_{\mathrm{m}}$ and $b_{\mathrm{m}}$ are the gradient and y-intercept of the linear profile found in Figure 9a by plotting the gradient, $m$, of Eqn. (15) against the biaxiality. Similarly, $m_{\mathrm{b}}$ and $b_{\mathrm{b}}$ are the gradient and y-intercept of the linear profile found in Figure $9 \mathrm{~b}$ by plotting the $y$-intercept, $b$, of Eqn. (15) against the biaxiality. Combining these with Eqn. (15) defines the proportionality parameter in terms of the biaxiality. 


$$
k_{\mathrm{c}}=\frac{m_{\mathrm{m}} B+b_{\mathrm{m}}}{\sqrt{\lambda_{\max }}}+\left(m_{\mathrm{b}} B+b_{\mathrm{b}}\right)
$$

In turn, this presents a new form of Eqn. (9) that is suitable for calculating the tearing energy in embedded flaws under multiaxial deformations.

$$
T=\frac{3}{2 \pi}\left(\frac{m_{\mathrm{m}} B+b_{\mathrm{m}}}{\sqrt{\lambda_{\mathrm{max}}}}+\left(m_{\mathrm{b}} B+b_{\mathrm{b}}\right)\right) w_{\mathrm{c}} c
$$

For this particular case, the values of $m_{\mathrm{m}}, b_{\mathrm{m}}, m_{\mathrm{b}}$ and $b_{\mathrm{b}}$ define the tearing energy through Eqn. (19), where for simplicity it is useful to incorporate the $3 / 2 \pi$ term in the proportionality parameter such that the tearing energy is given by:

$$
T=\left(\frac{(0.884-2.486 B)}{\sqrt{\lambda_{\max }}}+(1.293 B+0.375)\right) w_{\mathrm{c}} c
$$

\section{Discussion}

The correlation between the tearing energy calculated from the FEA model and the tearing energy predicted through Eqn. (19) is compared in Figure 10. For each of the 6 loading modes, the tearing energy was calculated at 5 stretches, evenly spaced over the strain range. For example, the uniaxial data was evaluated over the following stretches; 1.4, 1.8, 2.2, 2.6 and 3.0. Furthermore, at each stretch the data was evaluated for each of the 9 crack lengths modelled, $0.1 \leq c \leq 0.3$. The combined data is presented in the figures and grouped to the different loading modes. The tearing energy values predicted from the equation correlate extremely well to those predicted by FEA, particularly considering that this is for multiple crack lengths, stretches and loading modes.

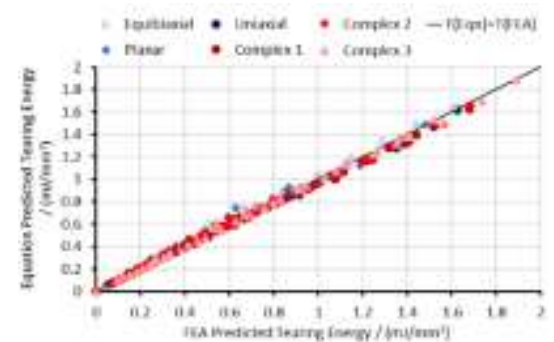

(a)

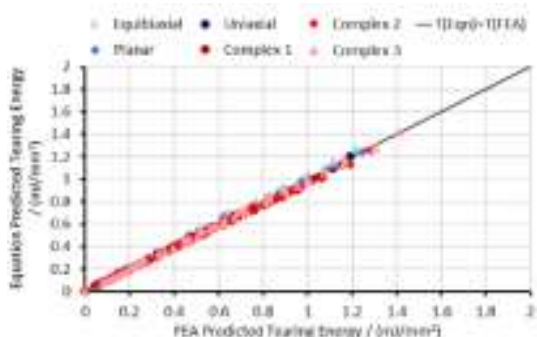

(b)

Fig.10. Correlation between tearing energy predicted through Eqn. (19) and by computational based fracture mechanics for the different loading modes for (a) NBR and (b) EPDM 
It is worth noting that the introduction of a proportionality parameter is ultimately an approximate solution. Rivlin and Thomas introduced the factor as a way to relate the far field energy to the local energy field about the crack - similar to the use of a stress intensity factor in LEFM. That said, capturing the proportionality parameter's load mode dependencies, as demonstrated in this work, is highly beneficial for applying theoretical fracture mechanics to more complex engineering components.

Calculating the tearing energy of a 3D flaw using a global approach also leads to an approximate solution, even when solidly based in fracture mechanics such as with the Energy Balance approach. Both the Energy Balance and heuristic equationbased approaches provide a single tearing energy value for the crack. In 3D cases, where the deformation of an initially circular crack geometry becomes an ellipsoid with 3 unique axes, the tearing energy is non-uniform around the crack's circumference. Nevertheless, a singular 'averaged' tearing energy value is likely a reasonable predictor of fatigue life, particularly when trying to optimise the design of an engineering component.

As explained in the introduction, the CED was developed to remove the need for a variable proportionality parameter whilst still allowing a de-rated tearing energy to be predicted from the far field parameters. Whilst CED had been demonstrated to work well on 2D cases, it had not been validated for 3D cases. The present study suggests that CED is still superior to SED in predicting fracture, but it works best with a proportionality parameter that is a function of the loading mode. In fact, CED appears to amplify the loading mode dependencies (see Figure 6), but this does not necessarily complicate the construction of a proportionality parameter that depends on the loading mode, particularly when the biaxiality definition in Eqn. (12) is used.

Other key findings from this paper are the linear relationships in Figure 9 that allow the proportionality parameter to be defined through the loading mode biaxialities. It is probable that these relationships define the geometric non-linearity of the crack and its influence on the tearing energy, or in other words, how the application of strain alters the crack tip geometry and tearing energy at the crack. There is likely a second non-linearity related to the material behaviour: how the rate of energy storage varies with loading mode. The gradients found by plotting Eqns. (16a) and (16b) may depend on the strain energy function and related model parameters, thereby controlling the material non-linearity of the fracture process. In this particular paper, the hyperelastic functions for both elastomers yielded similar profiles; hence, a single gradient sufficed. Future studies should investigate how the material models' parameters affect the gradient of these profiles, and determine whether they are indeed related to the material non-linearity aspect of the fracture process.

Finally, this work suggests that CED should be further explored with 3D simulations and testing. In particular, CED predicts fracture that is based on a single value for the tearing energy, whereas more generally the tearing energy can vary around a crack tip. To better assess the effect of this variation, future simulation 
work in 3D should use a local fracture mechanics approach at the crack tip to determine how maximum, minimum and average tearing energies relate to the CED.

\section{Conclusions}

Building on work in the 1920s, the first generally useful analytical model for the fracture of rubber was developed in the 1950s. It suggested that tearing energy could be deduced from crack geometry and far field strain energy that was released upon crack propagation. The model included a proportionality parameter, $k$, sometimes assumed constant, to scale the tearing energy in much the same way that stress concentration factors arise in the fracture of linear elastic solids. Early refinements of this model proposed specific forms of $k=k(\lambda)$, but only with partial success as proper accounting for the load mode dependence of rubber fracture was elusive. The introduction of cracking energy density was a significant breakthrough because it addressed loading mode sensitivities in a more general manner. It did so by scaling a system's total strain energy to the portion that was actually available to drive fracture. The model adopted a constant proportionality parameter from LEFM, but it has nevertheless been useful, especially for 2D analysis.

The present work provides a 3D finite element study of tearing energy and CED for an embedded flaw with multiaxial loads at finite strain. At a given stretch, tearing energy is found to be linearly related to crack length as expected. However, the proportionality parameter in the CED formulation is found to be variable, albeit in a predictable way. Using a definition of biaxiality that gives unique values for a given loading mode, the proportionality parameter becomes a linear function of biaxiality. This gives a simple analytical expression to relate tearing energy to CED, and it can be readily adopted for engineering design. Future work should extend this work to address: (i) deformations that are not pure, for instance simple shear; (ii) the effects of variation in tearing energy and geometry around the crack tip circumference; and (iii) the influence of the hyperelastic material model and related parameters.

Acknowledgements The authors would like to thank Schlumberger for funding and supporting this research. They would also like to thank Clwyd Compounders for providing the elastomers used. 


\section{References}

1. Inglis C.E. (1913) Stresses in a plate due to the presence of cracks and sharp corners. Trans. Inst. Nav. Archit. 55:219-239

2. Griffith A.A. (1921) The Phenomena of Rupture and Flow in Solids. Philos. Trans. R. Soc. A Math. Phys. Eng. Sci. 221:163-198

3. Rivlin R.S., Thomas A.G. (1953) Rupture of rubber. I. Characteristic energy for tearing. J. Polym. Sci. 10:291-318

4. Greensmith H.W. (1963) Rupture of rubber. X. The change in stored energy on making a small cut in a test piece held in simple extension. J. Appl. Polym. Sci. 7:993-1002

5. Lindley P.B. (1972) Energy for crack growth in model rubber components. J. Strain Anal. Eng. Des. 7:132-140

6. Lake G.J. (1970) Application of fracture mechanics to failure in rubber articles, with particulary reference to groove cracking in tyres. In: Int. Conf. Yield, Deformation and Fracture of Polymers. Cambridge: Plastics and Rubber Institute

7. Klüppel M., Huang G., Bandow B. (2008) Evaluation of Tearing Energy of Elastomer Materials. Kautschuk, Gummi, Kunststoffe. 61:656-659

8. Yeoh O.H. (2002) Relation between crack surface displacements and strain energy release rate in thin rubber sheets. Mech. Mater. 34:459-474

9. Mars W.V. (2006) Heuristic Approach for Approximating Energy Release Rates of Small Cracks Under Finite Strain, Multiaxial Loading. In: Elastomers and Components: Service Life Prediction - Progress and Challenges. $1^{\text {st }}$ Ed. Ed: V.A. Coveney. Woodhead Publishing. 91111

10. Zine A., Benseddiq N., Naït Abdelaziz M., Aït Hocine N., Bouami D. (2006) Prediction of rubber fatigue life under multiaxial loading. Fatigue Fract. Eng. Mater. Struct. 29:267-278

11. Ayoub G., Naït-Abdelaziz M., Zaïri F., Gloaguen J.M., Charrier P. (2011) A continuum damage model for the high-cycle fatigue life prediction of styrene-butadiene rubber under multiaxial loading. Int. J. Solids Struct. 48:2458-2466

12. Mars W.V., Fatemi A. (2005) Multiaxial fatigue of rubber: Part I: Equivalence criteria and theoretical aspects. Fatigue Fract. Eng. Mater. Struct. 28:515-522

13. Mars W.V., Fatemi A. (2006) Analysis of Fatigue Life under Complex Loading: Revisiting Cadwell, Merrill, Sloman, and Yost. Rubber Chem. Technol. 79:589-601

14. Ayoub G., Naït-Abdelaziz M., Zaïri F. (2014) Multiaxial fatigue life predictors for rubbers: Application of recent developments to a carbon-filled SBR. Int. J. Fatigue. 66:168-176

15. Poisson J.L., Méo S., Lacroix F., Berton G., Hosséini M., Ranganathan N. (2018) Comparison of Fatigue Criteria Under Proportional and Non-Proportional Multiaxial Loading. Rubber Chem. Technol. 91:320-338

16. Gough J., Muhr A.H. (2005) Energy Release Rates for Small Cracks in Rubber Components. In: Constitutive Models for Rubber IV: Proceedings of the Fourth European Conference on Constitutive Models for Rubber. $1^{\text {st }}$ Ed. Eds: P.-E. Austrell \& L. Kari. Taylor \& Francis Group London, UK. 51-57

17. Yeoh O.H. (2006) Strain Energy Release Rates for Some Classical Rubber Test Pieces by Finite Element Analysis. In: Elastomers and Components: Service Life Prediction - Progress and Challenges. $1^{\text {st }}$ Ed. Ed: V.A. Coveney. Woodhead Publishing. 75-89

18. Ogden R.W. (1972) Large Deformation Isotropic Elasticity - On the Correlation of Theory and Experiment for Incompressible Rubberlike Solids. Proc. R. Soc. A. Math. Phys. Eng. Sci. 326:565-584

19. Asare S., Busfield J.J.C. (2011) Fatigue life prediction of bonded rubber components at elevated temperature. Plast. Rubber Compos. 40:194-200

20. Wadham-Gagnon M., Hubert P., Semler C., Paidoussis M.P., Vezina M., Lavoie D. (2006) Hyperelastic modeling of rubber in commercial finite element software (ANSYS). Proceedings of the SAMPE '06: Creating New Opportunities for the World Economy. 